\title{
Las estrategias publicitarias de las marcas en el contexto de crisis
}

\author{
Elena Fernández Blanco \\ David Alameda García \\ Irene Martín Martín \\ Universidad Pontificia de Salamanca
}

\section{Palablas clave}

Crisis, marca, publicidad, estrategias, mensajes.

\section{Resumen}

La crisis económica ha evidenciado una situación que venía aconteciendo en la comunicación estratégica desde hace años: el debilitamiento del modelo publicitario establecido. Las organizaciones se enfrentan a una pérdida progresiva de la eficacia comunicativa de la publicidad; a la dificultad de conectar emocionalmente con sus consumidores; al retroceso de los medios convencionales; a la explosión de la era de las relaciones personales y a la hegemonía de un ciudadano, proactivo y crítico, que replantea el propio concepto de comunicación. Todo este contexto hace que las empresas y organizaciones redefinan sus estrategias publicitarias, modificando objetivos, públicos, valores, lenguajes, medios y modos de contactar con sus públicos.

El objetivo de este artículo es el estudio de las principales opciones estratégicas publicitarias que están desarrollando las organizaciones para sus marcas en el actual contexto de crisis. Para ello, se aborda el análisis de la estrategia publicitaria de una muestra significativa de los principales anunciantes del panorama nacional, representando a diferentes sectores de consumo. Mediante el análisis de contenido, combinado con análisis del discurso de las estrategias publicitarias de las principales marcas, procedemos al establecimiento de variables de análisis y a la categorización de los objetivos, estrategias, acciones y construcciones discursivas que las marcas desarrollan en sus políticas de comunicación. 
Los resultados de la investigación permiten concluir que las marcas están apostando por cuatro principales tipos de estrategias publicitarias: la estrategia de construcción de las U-brands, la defensa del valor de la marca tradicional, la estrategia centrada en el precio como principal argumento y la estrategia ofensiva de las marcas de distribuidor en torno a la calidad.

\title{
Advertising strategies of the brands in the context of crisis
}

\section{Key words}

Crisis, branding, advertising, strategies, messages.

\begin{abstract}
The economic crisis has shown a situation that had been happening on strategic communication for years: the undermining of the established advertising model. Organizations face a progressive loss of communicative effectiveness of advertising, to the difficulty of connecting emotionally with consumers, the decline of conventional media, to the explosion of the era of personal relations and the hegemony of a citizen, proactive, critical, that it questions the very concept of communication. All this context means that businesses and organizations to redefine their advertising strategies, changing objectives, public values, languages, ways and means to connect with their audiences.

The aim of this paper is the study of the major strategic choices that are developing advertising for their brands organizations in the current crisis. To do so, it addresses the analysis of the advertising strategy of a significant sample of the main advertisers on the national scene, representing various sectors of consumption. Through content analysis, discourse analysis combined with the advertising strategies of major brands proceed to the establishment of analytical variables and the categorization of the objectives, strategies, actions and discursive constructions that brands develop their communication policies.

The main results of the research are that makes betting on four main types of advertising strategies: the strategy of the U-brands, the defense of traditional brand equity, the strategy focused on price as their main argument and strategy offensive dealer marks around quality.
\end{abstract}

\section{Autores}

Elena Fernández Blanco [efernandezbl@upsa.es], David Alameda García [dalamedaga@upsa.es] e Irene Martín Martín [imartinma@upsa.es] son profesores en la Universidad Pontificia de Salamanca. 


\section{Introducción: la crisis y el consumo}

La situación económica mundial está teniendo efectos claros y significativos sobre la actitud de los consumidores españoles, hecho que se refleja en todos los mercados. El índice de confianza de los consumidores ha caído considerablemente, pasando a ocupar la economía una de las principales preocupaciones de los españoles. El origen de este escenario económico es global: subida del precio del dinero, incremento de la demanda de materias primas y energía de los países emergentes, alto precio del petróleo y burbuja inmobiliaria se sitúan como los principales motivos de la crisis (Stiglitz, 2010). El resultado es una contención generalizada de la demanda, un consumidor que se muestra más sensible a las variaciones de precios de los artículos de la cesta de la compra y un ascenso imparable de las marcas de los distribuidores, que han crecido el triple que las demás, a la vez que las grandes cadenas retiran referencias argumentando “escasa rotación”. Las principales consecuencias de la crisis para el consumo y las marcas son muy claras:

\subsection{Los hábitos de compra de los consumidores han cambiado}

En tiempos de crisis se inicia un periodo de incertidumbre y desconfianza en el que las familias y los individuos tienden a replantearse cómo distribuyen sus ingresos en previsión de tiempos peores. Esto les lleva, entre otras cosas, a realizar un replanteamiento racional sobre su consumo que sufre variaciones temporales o duraderas. Aquellos hábitos que los consumidores tengan más arraigados serán más difíciles de modificar o de eliminar (Cuneo, 2009:4). En este sentido, es la relación de los consumidores con las marcas y lo acostumbrados que estén a la presencia de una marca concreta durante un hábito de consumo lo que condiciona los posibles cambios. También la confianza es otro factor vital, ya que durante las crisis se producen menos cambios de marca dentro de una misma categoría de producto, no habiendo lugar a pruebas, salvo que éstas vayan dirigidas a probar un ahorro.

En este contexto, la imagen y la fuerza de las marcas cobra una importancia extrema, porque el debate que se produce en la mente del consumidor va más allá de un factor decisivo como el precio, y se sitúa, en algunos casos en otro nivel diferente: el de los hábitos de consumo.

Según Nielsen, el índice de confianza de los consumidores españoles se sitúa entre los países más pesimistas, con 12 puntos por debajo de la media mundial, lo que influye claramente en una reducción de las compras de mayor valor, y un cambio de actitud que les lleva a elegir los productos con mejor relación calidad-precio. En la actualidad, los españoles van más veces al supermercado, generando un ticket de compra de menor cantidad y dedicando más tiempo a mirar ofertas, a comparar. Las familias españolas reestructuran sus partidas de gastos, declarándose dispuestas a limitar al máximo o incluso eliminar gastos 
de peluquería, estética, ropa, calzado, equipamiento del hogar, ocio y consumo fuera del hogar. Otras partidas, como la gasolina, el teléfono móvil o el tabaco, las incluyen entre las que deben limitar sus gastos. Los gastos en vacaciones y en la cesta de la compra se incluyen entre los que deben organizarse; mientras otros se mantienen por ser considerados obligatorios, como los destinados a la salud, los gastos fijos del hogar como la luz y el agua, los impuestos, los seguros o la hipoteca. En definitiva, la reordenación del gasto familiar es una necesidad que están poniendo de relieve los propios consumidores (Nielsen, 2009). Estas mismas conclusiones se desprenden en el Informe Gestión de marcas en tiempos difíciles (TNS, 2010), cuando se afirma que nos encontramos ante un consumidor que investiga y busca más información antes de gastar y refuerza el control de su economía, estableciendo prioridades en sus gastos.

\subsection{Incremento de la marca del distribuidor}

La cuota de las marcas de distribución se ha incrementado un 2,7\% a finales de 2009 hasta alcanzar el 33\% del total de ventas de productos de gran consumo. Este dato asciende hasta el 39,4\% en el caso del sector de alimentación (Nielsen, Scantrends 2010). Las marcas blancas están penetrando con un gran éxito por diversos motivos, según Gabriela Salinas, consultora de Global Brand Manager en Deloitte (2009): existe una intensidad en la innovación; requieren una baja inversión publicitaria; hay una debilidad de las marcas que compiten en categoría; hay una buena relación calidad-precio, y existen diferencias de percepción de calidad. Además, las marcas blancas constituyen herramientas de negociación con los fabricantes de marcas. Sin embargo, para Roger (2010) los fabricantes han sido los propios responsables de la aparición y despegue de las marcas del distribuidor porque "representaron una oportunidad para aquellos fabricantes con capacidad ociosa (...), para soportar sus elevados costes fijos. Con el tiempo esta oportunidad se ha convertido en una amenaza para el conjunto de las marcas de fabricante que compiten en cada categoría”.

Independientemente de los factores, la actual coyuntura económica convierte a las marcas blancas en la mejor opción de ahorro para los consumidores, y desemboca en una consiguiente subida de su cuota de mercado, incluso en aquellos segmentos donde ya contaban con una alta participación, convirtiendo a España en el cuarto país de Europa con mayor cuota de mercado para la marca blanca ${ }^{1}$.

En definitiva, las marcas blancas han conseguido ganar la confianza de muchos consumidores basándose en dos pilares: el precio bajo y el declarado "buen origen” de los productos.

Sin embargo, es innegable que el fenómeno de las marcas blancas se ha visto incrementado por la estrategia de negocio de Mercadona, que añade a todo lo anterior el concepto de calidad. Mercadona rompe los esquemas sobre el tema

${ }^{1}$ Distribución 2009. Nuevos hábitos. En IPMARK, nº 710, 1-15 de noviembre 2008, pp. 7-8. 
de la valoración de la calidad que realizan los consumidores de las marcas, alcanzando un valor de calidad de la marca Hacendado, en ocasiones, superior a las marcas premium, según Innovex -Índice de innovación para marcas y empresas de Ipsos- (Vera, 2009:18).

Comprar por un menor precio se ha convertido en un valor en sí mismo: es la demostración de una compra inteligente, y muchos productos han perdido el valor diferencial de la marca. Para las marcas del fabricante esto plantea un dilema: competir por precio durante mucho tiempo no será sostenible en términos económicos y en términos de imagen les llevará a transmitir mensajes incoherentes que les puede arrastrar a perder su posicionamiento.

\subsection{La defensa del valor de marca}

Los cambios en los hábitos de los consumidores y el incremento del poder de las marcas blancas provocan una gran inestabilidad en los fabricantes de las grandes marcas, que ven como deben replantearse sus estrategias. El valor primordial de la marca y de lo que ésta supone para el consumidor continúa siendo para muchos anunciantes y profesionales del sector el activo fundamental a la hora de competir contra las marcas blancas.

En este sentido, se ha abierto un profundo debate sobre el valor de las marcas y su gestión. Ángel Riesgo (2009:18), que aboga por las marcas comerciales, considera que el poder de la distribución está rompiendo con la libre competencia. "Las marcas dan variedad a la oferta y la competencia permite que se multipliquen y compitan en atraer al consumidor, no sólo a través del precio sino de sus atributos racionales y emocionales". Incluso entiende que las marcas blancas están acabando con la innovación, ya que lo único que hacen es clonar, aprovechándose del éxito comercial de un nuevo formato, de un nuevo atributo o de una nueva formulación. "Sin innovación estamos matando la mitad del marketing, sólo el I+D+i y la publicidad pueden hacer crecer un producto de forma exponencial, sin innovación no hay futuro a medio plazo, tampoco para Mercadona” (Riesgo, 2009:18).

Las marcas blancas tienen un envase parecido al de los fabricantes, con un contenido idéntico y más barato, un producto que depende del original para existir y que ha absorbido su garantía, su innovación y su variedad. Por este motivo, las marcas premium se ven obligadas a replantearse su diferencial de precio de cara a la distribución y el consumidor (López, 2009:18). Sin embargo, la obtención y defensa de un premium razonable permite el marketing, la innovación, la calidad, la diferenciación de producto, la mejora continua, etc. No obstante, en épocas como la actual, donde el precio ha pasado a ser un factor importante dentro de esta crisis, la razón de premium, es decir aquello que explica y justifica el diferencial de precio, debe ponerse urgentemente sobre la mesa al mismo 
nivel de urgencia que la reestructuración de fábricas o el coste de las acciones de marketing.

En este contexto, las marcas comienzan a adoptar diferentes estrategias de defensa ante las marcas blancas ${ }^{2}$. Una de ellas es apostar por la mejora de la calidad para dar con una clara diferenciación. Otras acciones pasan por innovar con estrategias brillantes, luchar selectivamente, crear posiciones de valor ganador, e invertir en comunicación. Ese es precisamente el objetivo de esta investigación, intentar abordar las principales líneas comunicativas de las marcas en el contexto de crisis económica y ascenso imparable de las marcas de distribución ${ }^{3}$.

\subsection{Incremento de la actividad promocional en el punto de venta de todo tipo de formatos}

El principal beneficiado en este contexto es el propio consumidor, ya que puede ajustar sus necesidades de compra con mejores ofertas, precios más competitivos y en diferentes formatos que ofrece el mercado. Este cambio hacia lo promocional está motivado fundamentalmente por los propios cambios de hábitos de consumo ya que según el Wordpanel TNS “TNS LifeStyles 2008” el 77\% de las amas de casa utiliza cupones descuento, el 59\% participa en promociones de regalo de producto o el 44\% participa en promociones en etiquetas. Así, estamos acostumbrándonos que junto con la actividad publicitaria se inunden los establecimientos comerciales de actividades promocionales, descuentos especiales, semanas especiales, precios anti crisis u ofertas únicas.

El cambio en los hábitos del consumidor, el incremento de las marcas blancas, la defensa del valor de marca y el ascenso de las políticas promocionales son algunas de las principales consecuencias de la crisis que tienen su claro reflejo en las estrategias publicitarias de las marcas. Sin embargo, es imprescindible añadir que, al margen de la coyuntura de crisis, hoy la comunicación de las marcas atraviesa por un momento de renovación y adaptación a un nuevo modelo comunicativo, propugnado por la centralidad del consumidor que busca experiencias de marca más allá de los atributos de los productos. Este nuevo modelo de comunicación es reconocido y analizado por autores como Boschma (2008), Córdoba-Mendiola (2009), Deza (2008), Gobé (2005), Lenderman y Sánchez (2008), Martí y Muñoz (2008), Salmon (2008), Ollé y Riu (2008), Ros (2008), entre otros.

\footnotetext{
${ }^{2}$ En esta defensa del valor de la marca es interesante destacar la acción publicitaria conjunta realizada por la Asociación Española de Fabricantes de Productos de Marca (Promarca), Grupo Antena 3 y la agencia de publicidad Tapsa para frenar el desplazamiento del consumo hacia las marcas del distribuidor.

${ }^{3}$ Los principales resultados de esta investigación fueron presentados en el congreso "Comunicación y Desarrollo en la Era Digital”, Congreso Internacional AE-IC, Málaga, febrero 2010.
} 


\section{Metodología}

Tomando como referencia las consideraciones anteriores, la presente investigación se plantea con el objetivo principal de analizar las principales estrategias publicitarias de las marcas en el periodo actual de crisis. Se ha partido del análisis de un corpus de campañas publicitarias de algunas de las principales marcas comerciales (un total de 50 mensajes) en el medio televisión, entendiendo que a pesar del brusco descenso de inversión, sigue constituyendo el medio base de las planificaciones publicitarias para gran parte de los principales anunciantes de este país. Para ello, se han analizado las campañas más notorias y relevantes de 2009-2010 en relación al objeto de estudio, la crisis .

Por tanto, teniendo en cuenta que nuestros objetivos de análisis pretenden conocer las estructuras de significado que las empresas construyen en la comunicación publicitaria de sus marcas, así como los diferentes tipos de discurso que se emanan en sus mensajes, la aproximación cualitativa parece la más apropiada para acercarnos a la comprensión del fenómeno. No obstante, algunos de los resultados obtenidos se ofrecen de modo cuantificado para conocer el peso que tienen los diferentes relatos publicitarios relacionados con la crisis.

El modelo de análisis aplicado responde a un intento de simplificación del discurso publicitario de las marcas. Para ello, se han utilizado tres tipos de análisis que detallamos a continuación ${ }^{5}$ :

- Los valores de posicionamiento. Son aquellas categorías que definen el posicionamiento de la marca respecto al acontecimiento narrado; y que se observan por redundancia y saturación en los materiales analizados. Se trata de una concepción diferente a la tradición publicitaria (Joannis, 1992; Ries y Trout, 1989; Hernández, 1999), ya que en los procesos de construcción social de la realidad los valores de posicionamiento son nociones que ayudan a situarse a los sujetos frente a los objetos y acontecimientos.

- Los ejes discursivos. Por eje discursivo entendemos una forma amplia de concebir el concepto publicitario o eje (en términos de Joannis, 1992), es decir, aquel eje que organiza el mensaje de un anunciante hacia su target. Es un término mucho más amplio que el de concepto creativo, ya que se refiere a la manera de construir el relato publicitario y los principales argumentos discursivos utilizados por las marcas para comunicarse con sus públicos objetivo (ejes semánticos en la terminología de Barthes, 1964). Se trata, por tanto, de aquellos temas, argumentos y categorías que utilizan las marcas para organizar los mensajes.

\footnotetext{
${ }^{4}$ La selección del corpus de análisis se ha realizado en base a que hayan sido campañas premiadas en los diferentes certámenes publicitarios (El Sol, Cannes, etc.), así como las que han obtenido mayor repercusión y comentarios en la prensa especializada (Anuncios, Control de publicidad, El Publicista, El Periódico de la Publicidad), siempre en relación al marco de la crisis.

${ }^{5}$ Este modelo de análisis propuesto está basado en el diseño realizado por J. Benavides y aplicado al estudio de los mensajes publicitarios en varios de sus trabajos a partir de la tradición analítica del discurso, iniciada por Barthes y profundizada posteriormente por Greimas y otros autores. Véase, por ejemplo, Benavides, J. 2002a, 2002b y 2003.
} 
- Las estructuras de significado. Hace alusión a la organización significativa general que construyen las marcas respecto a los diferentes temas. Así, cada marca presente en la publicidad contribuye a crear esquemas de interpretación y refleja diferentes respuestas según el tipo de discurso utilizado.

- Todo este conjunto de categorías nos permiten sistematizar el contenido de los mensajes publicitarios para a partir de aquí inferir el conjunto de significaciones que se muestran en la publicidad desarrollada por las principales marcas. Los valores de posicionamiento nos permiten ir clasificando cada una de los relatos publicitarios en una categoría, y aunque se presentan mensajes que pueden ser adscritos a diferentes categorías de valores, se ha optado por clasificar la pieza publicitaria en función del valor de posicionamiento más predominante. Estos valores de posicionamiento nos remiten a un conjunto de ejes discursivos que de manera transversal organizan los relatos de las marcas. Por último, las estructuras de significado nos permiten entender las estrategias de comunicación que subyacen a estos discursos.

\section{Resultados de la investigación: el discurso publicitario de las marcas en contexto de crisis}

\subsection{Valores de posicionamiento de las marcas en relación a la crisis}

En el análisis efectuado se ha detectado la presencia de cuatro grandes categorías de valores de posicionamiento con los que las marcas construyen los marcos de referencia con sus públicos durante el periodo de recesión actual (ver Figura 1).

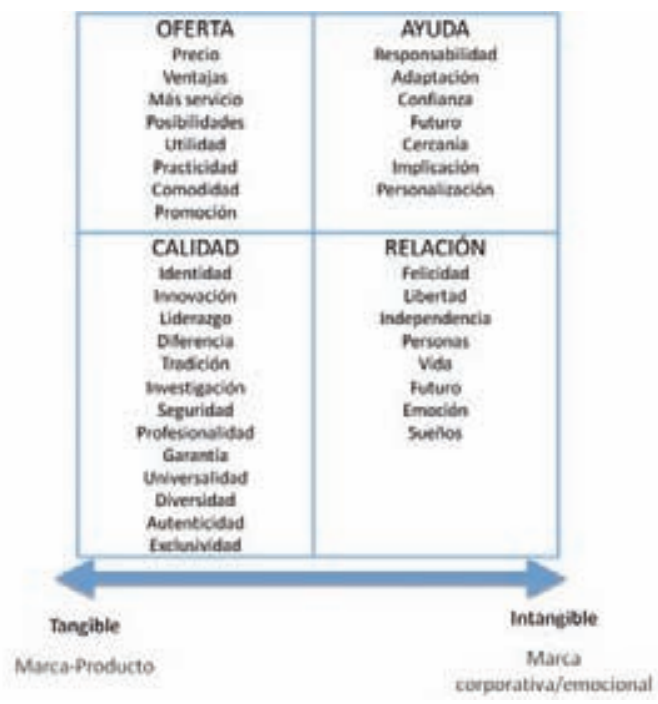

Figura 1. Principales valores de posicionamiento de las marcas en contextos de crisis. Fuente: elaboración propia. 


\subsubsection{Valores relacionados con la oferta}

El concepto de low cost se está asentando no sólo en la economía del día al día de los hogares españoles, sino también en las estrategias de comunicación de grandes marcas. Estrategias claramente contrarias al high cost que durante muchos años se han intentado justificar de mil maneras para que los consumidores pagaran más por lo que ahora saben que cuesta menos (Gil, 2009:27).

Por eso, hay una corriente de marketing que afirma que en situaciones de crisis lo que hay que hacer es aplicar el marketing en su esencia más profunda, satisfaciendo necesidades del consumidor por un precio movilizador y con un margen rentable (Alonso, 2008:20). Esta política de marketing tiene su reflejo directo en la comunicación, traduciéndose en mensajes de carácter informativo, relacionados con el producto, donde éste se posiciona como asequible y barato para el consumidor. El precio se convierte en el eje de la comunicación, por encima de los valores intangibles de marca y de empresa. Son mensajes, por tanto, donde se evidencia una ausencia de otros valores centrados en las cualidades del producto que permitan diferenciarlos en la esfera del consumo y pasa a primer plano el precio, las ventajas, el ahorro, el descuento, la comodidad de su adquisición, su practicidad o conveniencia.

Por ejemplo, Iberia ha ajustado el cinturón a sus precios y ha lanzado una agresiva y muy atractiva promoción a través de una campaña cercana que habla en un lenguaje que en estos momentos toca más el bolsillo que la fibra y capta la atención e interés de los consumidores. Carrefour también se sitúa en esta categoría de valores al afirmar que "Carrefour, te viene bien", con la campaña creada por la agencia Publicis. La agencia concibe una campaña que mueva al consumidor desde el precio de los productos, "porque bajamos el precio un $10 \%$ en una gran variedad de productos del pollo", "porque bajamos un $25 \%$ el precio de tus básicos en textil”, etc.

En el ahorro, encontramos también la campaña de ING Direct "Ya lo sabía" donde Cuenta naranja y ahorro son sinónimos; el spot de Renault "Ahórrate la crisis” y otras compañías áreas como Spanair y Air France, así como otras entidades bancarias como Barclays ("el hombre que aprendió a ahorrar").

\subsubsection{Valores relacionados con la ayuda}

El valor de posicionamiento centrado en la ayuda es un eje común de gran parte de las campañas publicitarias analizadas, porque ante la crisis, todas las marcas ofrecen colaboración y ventajas para sus clientes, ya sea a través de reducción directa de precio o aumento del servicio, las prestaciones y la calidad.

No obstante, con ayuda nos referimos concretamente a todo el conjunto de valores utilizados por aquellas marcas que trabajan para superar la crisis más allá del acto de consumo, es decir, marcas que se sitúan como referentes para ayu- 
dar a aquellos clientes que están pasando por dificultades. Rediseñan su oferta comercial para satisfacer las nuevas necesidades de ahorro y control del gasto ${ }^{6}$. A diferencia de los valores tangibles de precio de la categoría anterior, la marca adopta una postura de responsabilidad social ante la crisis, intentando mitigar sus efectos. Estamos no ante un discurso de producto, sino ante un discurso de marca corporativa donde la organización se presenta con una serie de atributos que posibiliten a sus públicos identificarlas como líder, desde la solidez y con vocación de ayuda y servicio.

Es el caso de Telefónica, compañía que adapta su oferta comercial para sintonizar con las nuevas necesidades de sus clientes, marcadas por el contexto económico desfavorable. A través de la campaña Te ayudamos, la medida más llamativa que ha anunciado es el descuento del 50\% que aplican a las facturas de sus clientes en paro durante todo este año. Asimismo, las pymes de nueva creación podrán acogerse a descuentos del 50\% en su primer año de vida, entre otros productos destinados a fomentar el ahorro y el control del gasto ${ }^{7}$.

En esta misma línea, BBVA con su campaña Destructores de paro, a cargo de la agencia Aftershare.com, ha emprendido una iniciativa para ayudar a autónomos y pymes a destruir el paro y crear futuro. Con esta campaña innovadora y cercana a los targets, BBVA se sitúa del lado de sus clientes y según reza el spot, ha conseguido resultados interesantes “...200.000 personas participantes, 40.000 curriculums recibidos y muchos empleos creados y muchos por crear. Para seguir adelante. BBVA”.

Otra entidad bancaria que se posiciona desde el lado de la ayuda y la preocupación es Banco Santander. A partir de un spot inscrito más en la línea emocional, Santander asume una gran responsabilidad: garantizar los ahorros de sus clientes o lo que es lo mismo "la confianza".

La Mutua Madrileña también es garantía y solidez para sus clientes, a través del claim "el poder de una gran compañía”. Esta marca se posiciona al lado de sus clientes y los hace partícipes de su ayuda de tal forma que éstos se "sienten" de la compañía ("soy” como afirma la canción que acompaña a la campaña).

Y El Corte Inglés en su vocación de servicio y responsabilidad para con sus clientes es el máximo exponente de esta preocupación, preparando y adaptando todo para la navidad: "Porque hay crisis y ha sido un mal año, te mereces unas buenas navidades. El Corte Inglés lo ha preparado todo para ti, como siempre”.

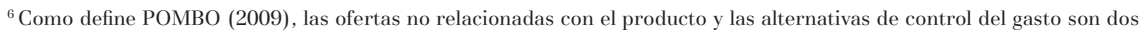
de las estrategias que las marcas utilizan para ayudar a sus clientes a sobrellevar la crisis.

7 “Telefónica apoya a sus clientes en paro” en Ipmark, n 719, 1-15 abril de 2009, p. 16.
} 


\subsubsection{Valores relacionados con la calidad}

En este grupo se incluyen las marcas que realizan una defensa clara de la calidad, tanto desde una posición de liderazgo y tradición, como desde el canal de distribución asociándose a buen precio. De este modo, la calidad se convierte en un valor común para las marcas premium y las marcas blancas; porque precisamente en épocas de crisis, las marcas blancas han ido ocupando progresivamente el territorio de la calidad, ocupado hasta ahora en exclusiva por las marcas de fabricante. Hoy, las marcas blancas suman la calidad a su indiscutible y sólido posicionamiento en precio. De ahí, que la lucha en el lineal se haya trasladado claramente al lenguaje publicitario, anulando o mitigando los valores de posicionamiento de marcas como Danone, Pascual, Fontaneda, Central Lechera Asturiana, Nescafé, etc., en torno a la calidad premium.

En esta pugna, las grandes marcas desde el paraguas de la calidad han apostado por diferenciarse del resto. Se trata de marcas que remarcan sus valores frente a la competencia o recuerdan al consumidor que no fabrican para otras marcas. Se ha convertido en una práctica publicitaria común insertar mensajes en los spots de televisión como "no fabricamos para otras marcas". Es el caso de Fontaneda Digestive, que pone de relieve las diferencias entre Fontaneda y otras marcas, al tiempo que se adjudican al producto valores de autenticidad, compromiso e innovación. Central Lechera Asturiana trabaja en la estrategia "no todas las leches son iguales" destacando los distintos atributos que convierten en única la leche Central Lechera Asturiana. Se transmite el mimo y cuidado con los que se trabajan en CLA, desde la ganadería hasta el envasado final, lo que le confiere una calidad y sabor especial, y se enfatiza la variedad de envases y formatos que se adaptan a las necesidades de cada uno ${ }^{8}$.

Leche Pascual también se ha sumado al grupo de grandes empresas que ha comenzado a informar a los consumidores de que no fabrican para otras marcas. Para ello, Leche Pascual ha encargado a la agencia S, C, P, F... la creación de un spot que, de una forma muy visual y utilizando la imagen de un brick, muestra cómo según se van anulando las ventajas y atributos diferenciales, así como los procesos que garantizan la calidad de Pascual.

Y otras grandes marcas como Danone, Nescafé o Mahou luchan por legitimar su calidad frente a su competencia y las marcas blancas. La marca de yogures y otros lácteos (Danonino, Actimel, Activia, etc.) está apostando fuertemente por este valor al manifestar rotundamente en sus piezas que es única y por eso no fabrica para otras marcas. En el caso de Nescafé es muy claro al afirmarse en su spot: "Superior en tueste, superior en sabor, superior en aroma y con toda la garantía de una gran marca”. Y para la marca de cervezas no vale con tomarse cualquier cerveza si UNA cerveza, con mayúsculas (como sueña el marinero en alta mar cuando llegue a tierra).

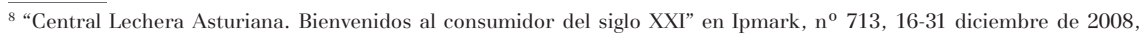
p.19. 
Frente al grupo de grandes marcas de fabricantes, también los mercados de bajo coste han lanzado en sus comunicaciones mensajes que resaltan la calidad de sus productos a un bajo precio. Así, Dia afirma de una manera muy gráfica que "si pagas más es porque quieres". Y para que quede claro nos pone un sencillo ejemplo de cómo un mismo producto aumenta su precio en el mercado según qué interferencias o extras incorpore en la cadena de su comercialización y presentación al consumidor. En clave de humor y sintiéndose ahora más fuertes, como todas las marcas que desde el principio han basado su posicionamiento en el precio, se atreve a contradecir la máxima de que la calidad tiene un precio. Del mismo modo, Lidl asegura que "la calidad no es cara" y recurre al origen de los productos, a la calidad de la materia prima. Su giro comunicativo toma como eje de campaña al director de la compañía, que diariamente selecciona los mejores productos directamente en origen ${ }^{9}$.

En definitiva, tras el análisis de las campañas, podemos establecer dos conceptos de calidad diferenciados, pero en competencia ante un mismo consumidor: la calidad como garantía de marca, autenticidad, legitimidad y superioridad, promulgada por las marcas premium y la calidad del producto, entendido desde su origen, su materia prima, lo natural, como clara estrategia ofensiva de las marcas del distribuidor. El primer grupo de mensajes representa un $73 \%$ del total de anuncios inscritos en esta categoría y el $27 \%$ restante corresponde a anuncios donde la calidad está asociada al producto.

\subsubsection{Valores en torno a la relación}

Otra estrategia adoptada por las marcas en este contexto es abandonar el escapismo y surrealismo, para ser sustituido por suaves campañas centradas en la felicidad de compartir y relacionarse con los demás (Pombo, 2009). La felicidad, no entendida desde el individuo, sino desde la comunidad. Precisamente ésta es la apuesta de posicionamiento de otras marcas, que siguiendo la línea emocional y de relación con el consumidor, se sitúan como compañeras de los consumidores para proporcionarles experiencias de marca o experimentar un branding emocional, como afirman Alloza (2001) y Gobé (2005), entre otros.

De este modo, los principales valores de posicionamiento están relacionados con la personalización, adaptación, ubicación de la marca en la esfera privada y personal del consumidor, la propuesta de un futuro mejor, los sueños, la seguridad e, incluso, la nostalgia. La marca pretende construir una relación afectiva a partir de una serie de valores emocionales, simbólicos e intangibles capaces de generar una experiencia positiva. La experimentación de la marca y de todo lo que ésta propone permite que el destinatario se sienta identificado, haga propias las propuestas que se argumentan en los mensajes y al final, se alcance la fidelidad, incluso en épocas de crisis.

9 “Lidl mejora su rentabilidad un 25\% con la nueva política de marca” en Expansión, 10/04/2010. 
Como claro ejemplo de lo que estamos comentando remitimos al caso de Coca Cola, donde a través de su campaña "Encuentro con la felicidad” descubrimos una emocionante historia de vida y felicidad. Nos invita a ser felices porque la vida es maravillosa, al margen de un momento de dificultad. Y de la misma compañía, Aquarius nos ofrece la posibilidad de soñar y nos invita a que persigamos nuestros sueños hasta el final, ya que "la vida es extraña y maravillosa y cada uno sueña lo que le da la gana”.

Este discurso minoritario viene también representado las diferentes campañas promovidas por las asociaciones sectoriales y medios de comunicación en defensa de la marca del fabricante, todo ello desde una visión relacional.

Estos cuatro tipos de valores de posicionamiento de las marcas apuntados giran en torno a dos referentes principales: la marca como soporte del producto y la marca como engagement. Los insights que hemos definido como oferta y calidad hacen referencia a atributos o cualidades tangibles de marcas que están amparando el producto que tienen detrás. Frente al aval del producto desde la marca, hay otro conjunto de marcas preocupadas por la adaptación y la relación y que recurren a lo intangible y a la esfera emocional.

Precisamente esta doble diferenciación de referentes nos hace ver cómo algunos anunciantes están volviendo a estrategias de marketing apoyadas en la clásica variable de producto para mantenerse en un mercado de crisis. Es la vuelta a lo tangible en la que la marca se define desde lo concreto, buscando la influencia del producto en las decisiones finales de compra. Así, grandes marcas como Danone deben descender al valor de producto para defender su valor de marca, apelando a calidad e investigación superiores a la competencia.

Sin embargo, otras marcas optan más por la vía de la personalización y lo emocional, independientemente del producto que respaldan, al ofrecer universos y escenarios de marca a los que el consumidor puede adscribirse (ver Figura 1).

Los mensajes publicitarios que presentan valores relacionados con la calidad y la oferta son los más predominantes en la muestra analizada, con un $30 \%$ sobre el total respectivamente (sumando ambas categorías el 60\% de la muestra analizada). Esta fuerte presencia tiene su explicación en el cambio de estrategias publicitarias de las marcas premium de alimentación que están apostando por poner en valor la calidad de sus productos y también, por el conjunto de mensajes enfocados a destacar el precio por encima de cualquier otro atributo en situaciones como la presente. Le sigue la categoría de valores relacionados con la ayuda (24\%). Y por último, los valores en torno a la relación son los más minoritarios, con una presencia del 16\% (Figura 2).

A nivel sectores a los que pertenecen las marcas analizadas en la investigación, es interesante poner de relieve que los valores relacionados con la oferta tienen su máxima presencia en el sector de la distribución y restauración, con un 40\% de los casos. Las marcas del distribuidor (MDD) aprovechan estos relatos para 
dar a conocer su precio competitivo. Finanzas y Transportes son los otros dos grandes sectores que apuestan por mensajes centrados en el precio de sus servicios, sobre todo, la guerra que se ha desatado en el sector bancario con las comisiones cero y la captación de nóminas ${ }^{10}$.

La categoría relacionada con la ayuda tiene su mayor presencia en los anuncios de las marcas de los grandes bancos y aseguradoras. Esta categoría, con un $66,6 \%$ de presencia, se explica por las campañas corporativas de los bancos, cajas de ahorro y compañías aseguradoras preocupadas por el bienestar de sus clientes, que quieren facilitarles su continuidad en la compañía. Desde el sector de la distribución, las campañas de El Corte Inglés suponen un 16\% de esta categoría de valores de posicionamiento reforzando su territorio de marca centrado en la cercanía al consumidor y la personalización.

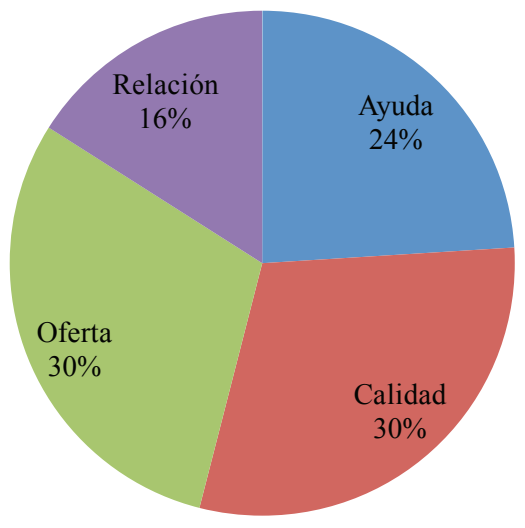

Figura 2. \% de campañas según valores de posicionamiento. Fuente: elaboración propia.

La calidad domina los mensajes del sector de la alimentación, con un $60 \%$ de presencia. Las grandes marcas de alimentación consolidadas y reputadas como Danone, Fontaneda, Nescafé, Pascual, etc. acaparan esta tipología de valores de posicionamiento. No obstante, también destaca en esta categoría el sector de la distribución $(26,6 \%)$.

Por último, los valores en torno a la relación tienen un nivel de presencia menor en la muestra analizada. Estos se construyen desde algunas marcas de bebidas y campañas corporativas de servicios privados a favor de las marcas (25\% y $75 \%$ respectivamente) (Figura 3).

\footnotetext{
10 "Las nóminas desatan la guerra publicitaria entre los bancos" en El Periódico de la publicidad, nº 313, 13 al 20 de marzo de 2009, p. 27.
} 


\subsection{Principales ejes discursivos}

Estos valores de posicionamiento nos remiten a tres tipos de ejes de significados o discursos que se entremezclan en los relatos publicitarios actuales (figura 4):

Figura 3. Valores de posicionamiento por sectores. Fuente: elaboración

\begin{tabular}{|c|c|c|}
\hline Valores & Sector & Total \\
\hline \multirow[t]{6}{*}{ Oferta } & Automoción & $6,7 \%$ \\
\hline & Distribución & $40,0 \%$ \\
\hline & Finanzas & $26,7 \%$ \\
\hline & Telecomunicaciones & $6,7 \%$ \\
\hline & Transporte & $20,0 \%$ \\
\hline & Total & $100,0 \%$ \\
\hline \multirow[t]{5}{*}{ Ayuda } & Distribución & $16,7 \%$ \\
\hline & Finanzas & $66,7 \%$ \\
\hline & Óptica & $8,3 \%$ \\
\hline & Telecomunicaciones & $8,3 \%$ \\
\hline & Total & $100,0 \%$ \\
\hline \multirow[t]{5}{*}{ Calidad } & Alimentación & $60,0 \%$ \\
\hline & Bebidas & $6,7 \%$ \\
\hline & Belleza e higiene & $6,7 \%$ \\
\hline & Distribución & $26,7 \%$ \\
\hline & Total & $100,0 \%$ \\
\hline \multirow[t]{3}{*}{ Relación } & Bebidas & $25,0 \%$ \\
\hline & Servicios privados & $75,0 \%$ \\
\hline & Total & $100,0 \%$ \\
\hline
\end{tabular}

\subsubsection{El discurso de la ayuda desde el ahorro y desde la responsabilidad social.}

Es el de mayor presencia en la muestra analizada y es utilizado por la mayor parte de las marcas. Está centrado en la colaboración ofrecida a los consumidores en épocas de crisis. Son relatos que promocionan el ahorro para seguir adquiriendo productos con la misma calidad, servicios y prestaciones a un precio más barato. Y también, son mensajes en los que la marca es una ayuda y colaboración para el consumidor.

Este discurso tiene como principal referente al producto y las ventajas económicas que reúne para el consumidor. El mensaje se articula como una transmisión de información donde el ahorro, ayuda y las ventajas económicas pasan a ser los protagonistas del relato.

En relación al tipo de comunicación suelen ser mensajes muy directos, informativos, dónde se explica cuál es este ahorro y qué ventaja tiene para el consu- 
midor y muchos de ellos se construyen desde el humor, situaciones divertidas y exageraciones (al mal tiempo, buena cara). La predicación publicitaria es la función fundamental que observamos en este tipo de discurso, en el que los significados que se transmiten para definir y diferenciar a la marca frente a la competencia están centrados en especificaciones en torno al precio y el ahorro y también, la necesidad social y económica que cubren en el mercado.

Aún así, cuando el discurso se centra en la ayuda y adaptación al consumidor para superar la crisis se utiliza ya un tipo de comunicación más personal, simbólica y que busca la empatía con sus consumidores.

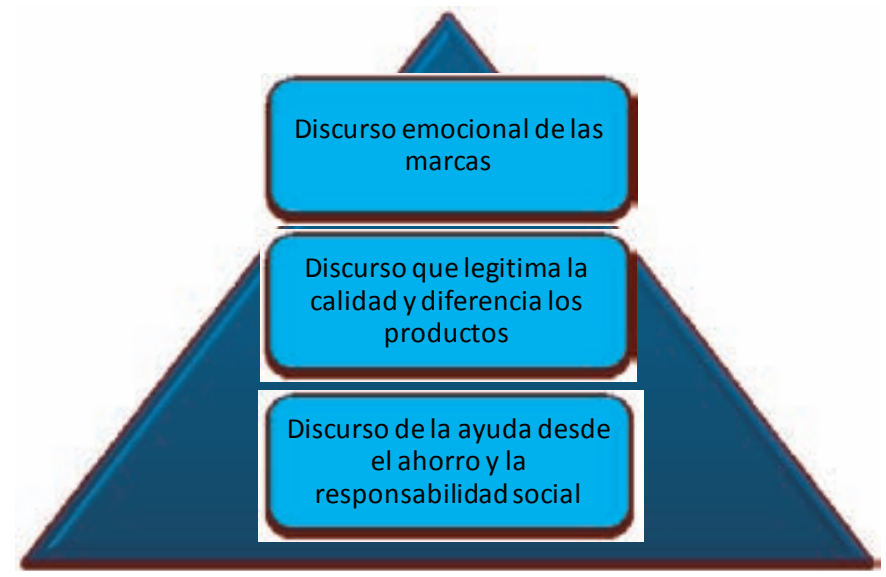

Figura 4. Principales ejes discursivos. Fuente: elaboración propia.

\subsubsection{El discurso que legitima la calidad y diferenciación de los productos}

Se trata del conjunto de rasgos y variables que se introducen en la descripción del producto o servicio y cuyo principal fin es demostrar que los productos son de calidad y por tanto, diferentes a sus competidores. Esta tipología discursiva es utilizada tanto por marcas del fabricante como por marcas del distribuidor. Mientras que las primeras se centran en rasgos que relacionan la calidad con la tradición y la legitimidad de sus productos en el mercado, las segundas centran el discurso en la calidad entendida como buenos productos porque son seleccionados en origen, todo ello a buen precio.

De este modo, la marca (independientemente del tipo) aporta información sobre el producto o servicio al señalar su calidad. Pero no sólo lo identifica, sino que también cumple con la función referencial cuando la marca ofrece un conjunto de rasgos sobre el producto, ayudando así al consumidor a identificarse y concentrarse sobre una oferta que responda mejor a sus necesidades específicas. Al tiempo, la marca está cumpliendo una tercera función, la de garantía, al 
ofrecer al consumidor un compromiso público de calidad, rendimiento, prestaciones y seguridad del producto al que ampara. Estas tres funciones básicas de una marca: identificación, referencia y garantía están siendo asumidas también en las estrategias de marketing y de comunicación de las marcas blancas, por lo que desplazan a las marcas del fabricante y comparten los mismos escenarios.

El centro del relato es la marca aunque como un paraguas del producto que hay detrás. El tipo de comunicación es muy demostrativa y racional, aunque algunos apelan a lo emocional para primar la calidad del producto sobre otras marcas secundarias (marcas de toda la vida, únicas, de prestigio). Los programas de comparación (metáforas y analogías) son muy recurrentes precisamente para evidenciar la calidad de la marca.

\subsubsection{EI discurso emocional de las marcas}

Hablamos del discurso de aquellas marcas que entran en la esfera de la personalización y la atención de otro tipo de necesidades más simbólicas y emocionales. La marca se inscribe en la esfera personal de los públicos, forma parte de sus realidades cotidianas, ofrecen posibilidades, futuro y personalización. Precisamente este discurso, minoritario en el conjunto de la muestra analizada, explica que los consumidores sigamos teniendo marcas concretas en nuestro ámbito de consumo para determinadas categorías de producto sin importar su precio o la crisis, sino simplemente por lo que nos proporciona desde su vertiente más emocional y simbólica.

El protagonista de los mensajes deja de ser el propio producto o la marca que lo soporta y toma el relevo el propio destinatario de la comunicación, el que puede vivir la experiencia de marca. En esta línea, la comunicación está basada más en la emoción y en los sentimientos, busca la vinculación afectiva entre la marca y el consumidor, mediante contenidos dirigidos a sus sentidos (alegría, felicidad, posibilidades, anhelos, sueños...). Las historias reales de la gente ganan terreno frente a los mensajes anodinos de productos a los que nos tiene acostumbrados la publicidad.

\section{Conclusiones}

Tras el análisis de los discursos publicitarios de las marcas ante este contexto de cambio motivado por la crisis pero también ante el agotamiento del modelo tradicional de publicidad, podemos afirmar que estos relatos responden a cuatro estructuras de significado distintas en torno al tema de la crisis y que conviven en las prácticas publicitarias televisivas. Cada una de estas estructuras refleja, en el fondo, una manera de articular y concebir la estrategia de comunicación y marketing para las marcas. Y estas estructuras de significado se han obtenido 
en base a la saturación y redundancia de los valores de posicionamiento y ejes analizados en los mensajes que han constituido la muestra de estudio:

- La construcción de las U-brands. Se detecta una estrategia comunicativa encaminada a la generación de engagement (Martí y Muñoz, 2008), de conexión emocional con el consumidor. La marca deja de ser responsabilidad de las empresas y organizaciones, para ser construida por el usuario, que va generando su experiencia a través de diferentes relaciones con medios masivos, directos, personales, interactivos y también con otros usuarios. Un consumidor más activo, crossumer y más exigente frente a la oferta.

- La defensa del valor de la marca tradicional. Otro grupo importante de organizaciones, generalmente los líderes en sus sectores, abordan estrategias de marketing y comunicación defensivas, intentando proteger los valores de la marca conseguidos a lo largo del tiempo. Se pone en valor la marca de toda la vida frente a marcas inferiores en la categoría e incluso frente a las marcas del distribuidor. El objetivo es construir o mantener un posicionamiento de superioridad, con unos valores de marca relacionados con el prestigio, la autenticidad, la tradición y la mayor notoriedad en el mercado.

- La estrategia centrada en el precio como principal argumento. Marcas de diferentes sectores de consumo deciden aprovecharse de la crisis y convertirla en una oportunidad. Encontramos así acciones de comunicación y mensajes de empresas y organizaciones centradas en comunicar precio, rebajas, "precios anti-crisis", ventajas, etc. Pero además, otras marcas aprovechan esta coyuntura para posicionarse más cerca del consumidor como marcas que ayudan y se responsabilizan de ofrecer lo mejor a sus clientes.

- La estrategia ofensiva de las marcas de distribuidor. Las marcas de distribuidor, e incluso marcas que trabajan bajo el concepto de "low cost", aprovechan la coyuntura económica para construir el valor de la calidad, intentando arrebatar así el posicionamiento de las grandes marcas comerciales. Apuestan por una opción estratégica centrada en la comunicación del producto que distribuyen y su calidad.

\section{Referencias}

Alloza, Ángel (2001). La gestión estratégica de la marca. En Villafañe, Justo. El estado de la publicidad y el corporate en España y Latinoamérica. Informe Anual. Madrid: Pirámide, pps. 207-269.

Alonso, José Miguel (2008). La hora de la verdad. Estrategias de marketing para tiempos de crisis. En: Anuncios, no 1251, 15 al 21 de septiembre, pp. 20-21.

Barthes, Roland (1964). Réthorique de l'imagen. En: Comunications, $\mathrm{n}^{\circ}$ 4, París: Seuil. 
Benavides, Juan (2002a). Los valores, los discursos y el conocimiento en el ámbito de la nueva cultura publicitaria y audiovisual. En: Comunicación y Estudios Universitarios, $\mathrm{n}^{0}$ 11, Valencia: Universidad Cardenal Herrera-CEU, pp. 105-117 Benavides, Juan (2002b). La publicidad televisiva en el año 2001: el cambio en los valores de marca. En: Villafañe, Justo. (dtor). El estado de la publicidad y el corporate en España y Latinoamerica. Madrid: Pirámide, pp. 72-81.

Benavides, Juan (2003). Los valores emergentes en la publicidad televisiva durante el año 2002. En: Villafañe, Justo (dtor). El estado de la publicidad y el corporate en España y Latinoamerica. Madrid: Pirámide, pp. 67-79.

Boschma, Jeroen (2008). Generación Einstein. Más listos, más rápidos y más sociables. Comunicar con los jóvenes del siglo XXI. Barcelona: Gestión 2000.

CÓRDOBA-MENDIOLA, Daniel (2009): Coolhunting. Cazar y gestionar las tendencias y las modas que mueven el mundo. Barcelona: Gestión 2000.

Cuneo, Álvaro (2009). Las marcas en tiempos de crisis. En: El Periódico de la Publicidad, $\mathrm{n}^{\mathrm{o}}$ 295, 16 al 23 de enero, p. 4.

Deza Pulido, Mónica (2008). Consumidores Nómadas. El siglo del mobile marketing. La Coruña: Netbiblo.

Gil Carrasco, Óscar (2009). Low cost for president. En: IPMARK, nº 716, 16-28 febrero, p. 27.

Gobé, Marc (2005). Branding emocional. El nuevo paradigma para conectar las marcas emocionalmente con las personas. Barcelona: Divine Egg.

Hernández, Caridad (1999): Manual de creatividad publicitaria. Madrid: Síntesis.

IPMARK (2008). Distribución 2009. Nuevos hábitos. En IPMARK, n ${ }^{0}$ 710, 1-15 noviembre 2008, pp. 7-8.

Joannis, Henri (1992). El proceso de creación publicitaria. Bilbao: Deusto.

Lenderman, Max y Sánchez, Raúl (2008). Marketing experiencial. La revolución de las marcas. Madrid: ESIC.

López, Nicolás (2009). Premium. En: IPMARK, no 716, 16-28 febrero, p. 18.

Martí, José y Muñoz, Pablo. (2008). Engagement marketing. Madrid: Prentice.

Nielsen (2009). Estudio Nielsen Online Consumer Confidence and Opinion.

Nielsen (2010). Nielsen Scantrends, enero 2010.

Ollé, Ramón y RIU, David (2008). El nuevo Brand Management. Barcelona: Gestión 2000. 
Pombo, Sandra (2009). Recession: Friend or Foe? A review of the strategies and tactics used to communicate in a downturn. En: Communicating in a downturn. Reccesionary marcoms tactics \& strategies. Xtreme Insight, Special report. Disponible en www.xtremeinformation.com/resources/recession.pdf, consultado el 08/11/2009.

Ries, Al y Trout, Jack (1989). Posicionamiento. Madrid: McGraw Hill.

Riesgo, Ángel (2009). Marcas negras. En: IPMARK, no 719, 1-15 abril, p. 18.

Roger, Guillermo (2010). Las marcas distribuidor en el sector de la alimentación. En: EAE Business School, Strategic Research Center. Disponible en http://www.scribd.com/doc/32143173/Las-Marcas-de-Distribuidor-en-Alimentacion-Guillermo-Roger-Mayo-2010, consultado el 4/6/2010.

Ros, Vicente (2008). e-branding. Posiciona tu marca en la red. A Coruña: Netbiblo.

Salinas, Gabriela (2009). La amenaza de las marcas blancas: ¿cómo competir efectivamente con este segmento cada vez más amplio y diverso?. En: I Foro sobre Comunicación y Marketing, McCann Erickson/El Periódico de la Publicidad, abril 2009.

Salmon, Christian (2008). Storytelling. La máquina de fabricar historias y formatear mentes. Barcelona: Península.

Stiglitz, Joseph E. (2010). Caída libre. El libre mercado y el hundimiento de la economía mundial. Madrid: Taurus.

TNS (2008). Wordpanel TNS. En: TNS LifeStyles 2008. Madrid: TNS.

TNS (2010). Gestión de marcas en tiempos difíciles. Madrid: TNS.

Vera, Antonio (2009). Mercadona, la enseña de distribución más innovadora. En: IPMARK, n ${ }^{\mathrm{o}}$ 721, 1-15 mayo 2009, p. 18.

\section{Referencia de este artículo}

Fernández Blanco, Elena; Alameda García, David y Martín Martín, Irene (2011). Las estrategias publicitarias de las marcas en el contexto de crisis. En: adComunica. Revista Científica de Estrategias, Tendencias e Innovación en Comunicación, $\mathrm{n}^{\mathrm{o}}$ 1. Castellón: Asociación para el Desarrollo de la Comunicación adComunica, Universidad Complutense de Madrid y Universitat Jaume I, 119-138. 